\title{
A GEOMETRICAL CHARACTERIZATION FOR THE AFFINE DIFFERENTIAL INVARIANTS OF A SPACE CURVE
}

\author{
L. A. SANTALó
}

1. Introduction. Let $x=x(s)$ be the vector equation of a space curve $C$ with the affine arc length $s$ as parameter. It is known that $x(s)$ satisfies a differential equation of the following form $[1, \text { p. } 73 ; 3, \text { p. 76 }]^{1}$

$$
x^{\prime \prime \prime \prime}+k x^{\prime \prime}+t x^{\prime}=0,
$$

where the primes represent derivatives with respect to $s$. The vector $x^{\prime}$ is the tangent vector and the vectors $x^{\prime \prime}$ and $x^{\prime \prime \prime}$ are called the affine principal normal and the affine binormal, respectively, of the curve $C$ at the point considered. The vectors $x^{\prime}, x^{\prime \prime}, x^{\prime \prime \prime}$ with the initial point at the point $x$ of the curve $C$ constitute the affine fundamental trihedral at $x$ and they satisfy the following relation $[1$, p. 72 ; 3, p. 78]

$$
\left(x^{\prime}, x^{\prime \prime}, x^{\prime \prime \prime}\right)=1 \text {. }
$$

The plane determined by the point $x$ and the edges $x^{\prime}, x^{\prime \prime}$ of the affine fundamental trihedral is the osculating plane at $x$; the plane determined by $x$ and the edges $x^{\prime \prime}, x^{\prime \prime \prime}$ is the affine normal plane and the plane determined by $x$ and the edges $x^{\prime}, x^{\prime \prime}$ is the affine rectifying plane of the curve $C$ at the point $x$.

Sometimes it is convenient to use the vector $k x^{\prime} / 4+x^{\prime \prime \prime}$ which is called the binormal of Winternitz [1, p. 76]. The invariants $k$ and $t$ (functions of the affine arc length $s$ ) are called the affine curvature and the affine torsion respectively.

For the affine fundamental trihedral and for $k$ and $t$ some geometrical characterizations have been given by Blaschke [1, chap. 3], Salkowski [3, p. 76] and Haack [2]. The purpose of the present paper is to give a new geometrical construction for the mentioned elements, which we believe is simpler than those previously obtained.

2. Geometrical elements associated to an ordinary point of a space curve. Let us consider the space curve $C$ represented by the vector equation $x=x(s)$ ( $s=$ affine arc length) in the neighborhood of the point $x_{0}=x(0)$. If we denote by $x_{0}{ }^{(i)}$ the derivatives $d^{(i)} x / d s^{i}$ at the point $s=0$, since $x_{0}^{\prime}, x_{0}^{\prime}, x_{0}^{\prime \prime \prime}$ are not coplanar (by (1.2)), any point $y$ of the space can be expressed in the form

Received by the editors January 17, 1946.

${ }_{1}^{1}$ Numbers in brackets refer to the references cited at the end of the paper. 


$$
y=x_{0}+\xi_{1} x_{0}^{\prime}+\xi_{2} x_{0}^{\prime \prime}+\xi_{3} x_{0}^{\prime \prime \prime}
$$

where $\xi_{1}, \xi_{2}, \xi_{3}$ may be considered as the coordinates of the point $y$ with respect to the affine fundamental trihedral.

For the points $x(s)$ of the curve $C$ we have

$$
x(s)=x_{0}+s x_{0}^{\prime}+\frac{1}{2} s^{2} x_{0}^{\prime \prime}+\frac{1}{3 !} s^{3} x_{0}^{\prime \prime}+\frac{1}{4 !} s^{4} x_{0}^{\prime \prime \prime}+\cdots
$$

and taking into account the relation (1.1) we obtain

$$
\begin{aligned}
& \xi_{1}=s-\frac{1}{4 !} t s^{4}-\frac{1}{5 !} t^{\prime} s^{5}+\cdots, \\
& \xi_{2}=\frac{1}{2} s^{2}-\frac{1}{4 !} k s^{4}-\frac{1}{5 !}\left(k^{\prime}+t\right) s^{5}+\cdots, \\
& \xi_{3}=\frac{1}{3 !} s^{3}-\frac{1}{5 !} k s^{5}+\cdots,
\end{aligned}
$$

where $k^{\prime}=d k / d s, t^{\prime}=d t / d s$.

Let us now consider the following geometrical elements associated to the curve $C$ at the point $x_{0}$ :

(a) The quadric cone $K$. By $K$ we denote the quadric cone determined by the tangent of $C$ at the point $x_{0}$ and the parallel lines through $x_{0}$ to the tangents of $C$ at four neighboring points as each of these points independently approaches $x_{0}$ along $C$. This quadric cone $K$ has been considered by Haack [2] and its equation in terms of the coordinates $\xi_{i}$ is $[2$, p. 159]

$$
\xi_{2}^{2}+k \xi_{3}^{2}-2 \xi_{1} \xi_{3}=0 .
$$

(b) The osculating cubical parabola $Q$. A twisted cubic which has the plane at infinity as osculating plane is called a cubical parabola. The parametric equations of a cubical parabola have the following form

$$
\xi_{i}=a_{i}+b_{i} u+c_{i} u^{2}+d_{i} u^{3} \quad(i=1,2,3) .
$$

In order to find the cubical parabola which has contact of the highest order with the curve $C$ at the point $x_{0}$, let us put

$$
s=u+m_{1} u^{2}+m_{2} u^{3}+m_{3} u^{4}+\cdots
$$

in the equations (2.2). We obtain

$$
\xi_{1}=u+m_{1} u^{2}+m_{2} u^{3}+\left(m_{3}-\frac{1}{4 !} t\right) u^{4}+\cdots,
$$




$$
\begin{aligned}
& \xi_{2}=\frac{1}{2} u^{2}+m_{1} u^{3}+\left(m_{2}+\frac{1}{2} m_{1}^{2}-\frac{1}{4 !} k\right) u^{4}+\cdots \\
& \xi_{3}=\frac{1}{3 !} u^{3}+\frac{1}{2} m_{1} u^{4}+\cdots
\end{aligned}
$$

If we take the values

$$
m_{1}=0, \quad m_{2}=\frac{1}{4 !} k, \quad m_{3}=\frac{1}{4 !} t
$$

we see that the cubical parabola

$$
\xi_{1}=u+\frac{1}{4 !} k u^{3}, \quad \xi_{2}=\frac{1}{2} u^{2}, \quad \xi_{3}=\frac{1}{3 !} u^{3}
$$

is the only cubical parabola which has a fourth order contact with the curve $C$ at the point $x_{0}$. Consequently the equations (2.6) are the parametric equations of the osculating cubical parabola $Q$ of $C$ at $x_{0}$.

The quadric cone $K$ and the twisted cubic $Q$ are affinely connected to the curve $C$ at the point $x_{0}$. We shall use also the following cone $K^{*}$ which is projectively connected with $C$.

(c) The osculating quadric cone $K^{*}$. With $K^{*}$ we denote the unique quadric cone with its vertex at the point $x_{0}$ which has seven-point contact with $C$ at the point $x_{0}$. In order to find the equation of $K^{*}$ let us substitute the expansions (2.2) in the general quadratic equation

$$
A \xi_{1}^{2}+B \xi_{2}^{2}+C \xi_{3}^{2}+D \xi_{1} \xi_{2}+E \xi_{1} \xi_{3}+F \xi_{2} \xi_{3}=0
$$

and then, demanding that this equation be satisfied identically in $s$ as far as the terms in $s^{6}$, we find

$$
A=D=F=0, \quad 3 B+2 E=0, \quad 10 C-15 k B-3 k E=0 .
$$

Hence, the osculating quadric cone $K^{*}$ is given by the equation

$$
20 \xi_{2}^{2}+21 k \xi_{3}^{2}-30 \xi_{1} \xi_{3}=0 .
$$

3. Geometrical characterization of the affine fundamental trihedral of a space curve at an ordinary point. From (2.6) we deduce that the straight line which connects the point $x_{0}$ of the curve $C$ with the point at infinity of the osculating cubical parabola $Q$ at $x_{0}$ has the direction of the vector

$$
\frac{1}{4} k x_{0}^{\prime}+x_{0}^{\prime \prime \prime}
$$


that is, coincides with the binormal of Winternitz ( $\$ 1)$.

The plane determined by this binormal and the tangent line of $C$ at $x_{0}$ is the affine rectifying plane and the polar line of this affine rectifying plane with respect to the cone $K$ turns out to be the affine principal normal line.

If we let $\xi_{2}=0$ in the equation (2.3) we find that the intersection of the affine rectifying plane with the quadric cone $K$ is composed of the tangent line and of the straight line given by the equations $\xi_{2}=0$, $k \xi_{3}-2 \xi_{1}=0$, that is, the line which has the direction of the vector $k x_{0}^{\prime} / 2+x_{0}^{\prime \prime \prime}$.

Since the vectors $x_{0}^{\prime}, k x_{0}^{\prime} / 4+x_{0}^{\prime \prime \prime}$ and $k x_{0}^{\prime} / 2+x_{0}^{\prime \prime \prime}, x_{0}^{\prime \prime \prime}$ are harmonically separated it turns out that the affine binormal is the line harmonic conjugate to the intersection line (different from the tangent) of the cone $K$ with the affine rectifying plane with respect to the tangent line and the binormal of Winternitz. The plane determined by the affine binormal and the affine principal normal is the affine normal plane. Thus the following theorem is established:

At an ordinary point $x_{0}$ of a space curve $C$ let us consider the osculating cubical parabola $Q$ and the cone $K$ defined in $\$ 2$. The direction of the point at infinity of $Q$ gives the binormal of Winternitz of $C$ at $x_{0}$. This binormal determines with the tangent line the affine rectifying plane, whose polar line with respect to the cone $K$ is the affine principal normal of $C$ at $x_{0}$. The harmonic conjugate of the intersection line (different from the tangent) of $K$ with the affine rectifying plane with respect to the pair formed by the tangent line and the binormal of Winternitz is the affine binormal line of $C$ at $x_{0}$.

Instead of the cone $K$ and the cubical parabola $Q$ in order to give a geometrical characterization of the affine fundamental trihedral connected with the space curve $C$ at the point $x_{0}$, we can use only the cones $K$ and $K^{*}(\$ 2)$. For this purpose let us seek the straight line which passes through the point $x_{0}$ and has the same polar plane with respect to both cones $K$ and $K^{*}$. If we use the coordinates $\xi_{1}, \xi_{2}, \xi_{3}$ of (2.1) the polar plane with respect to $K$ of the line which connects $x_{0}$ with the point $\xi_{1}{ }^{0}, \xi_{2}{ }^{0}, \xi_{3}{ }^{0}$ is given by the equation

$$
\xi_{3}^{0} \xi_{1}-\xi_{2}^{0} \xi_{2}-\left(k \xi_{3}^{0}-\xi_{1}^{0}\right) \xi_{3}=0
$$

and the polar plane of the same line with respect to the cone $K^{*}$ is

$$
15 \xi_{3}^{0} \xi_{1}-20 \xi_{2}^{0} \xi_{2}-\left(21 k \xi_{3}^{0}-15 \xi_{1}^{0}\right) \xi_{3}=0 .
$$

In order that the planes (3.1) and (3.2) coincide, we must have 
either $\xi_{2}{ }^{0}=\xi_{3}{ }^{0}=0$ or $\xi_{1}{ }^{0}=\xi_{3}{ }^{0}=0$. The first solution corresponds to the tangent line whose common polar plane with respect to the cones $K$ and $K^{*}$ is the osculating plane. The second solution corresponds to the affine principal normal whose polar plane with respect to the cones $K$ and $K^{*}$ is the affine rectifying plane. Then, upon excluding the tangent line, the affine principal normal is the only line which has the same polar plane with respect to the quadric cones $K$ and $K^{*}$.

We have seen that the affine rectifying plane intersects the cone $K$, excluding the tangent line, in the line $R$ of the vector $k x_{0}^{\prime} / 2+x_{0}^{\prime \prime \prime}$. Analogously we find that the affine rectifying plane intersects the cone $K^{*}$ besides the tangent in the line $R^{*}$ of the vector $7 k x_{0}^{\prime}+10 x_{0}{ }^{\prime \prime}$. Consequently the cross ratio of the tangent $T$, affine binormal $B$ and the lines $R$ and $R^{*}$ has the value

$$
\lambda=\left(T B R R^{*}\right)=\left(\infty, 0, \frac{1}{2} k, \frac{7}{10} k\right)=\frac{7}{5} .
$$

Thus we have obtained:

Let $K$ and $K^{*}$ be the quadric cones attached to the space curve $C$ at the point $x_{0}$ defined in $\$ 2$. The affine principal normal of $C$ at $x_{0}$ is the only line which has the same polar plane with respect to both cones $K$ and $K^{*} ;$ its common polar plane is the affine rectifying plane. Let $R$ and $R^{*}$ be the intersection lines, the tangents excluded, of the rectifying plane with the cones $K$ and $K^{*}$ respectively; the affine binormal is the line $B$ such that the cross ratio (TBRR*) has the value $7 / 5$.

4. Geometrical interpretation of the affine curvature. If we substitute the expressions (2.6) in (2.3) we find that the cubical parabola $Q$ has with the cone $K$ four coincident points in $x_{0}$ and two other intersection points corresponding to the values of $u$ satisfying the equation

$$
k u^{2}=6
$$

Let us put

$$
u_{1}=\left(\frac{6}{k}\right)^{1 / 2}, \quad u_{2}=-\left(\frac{6}{k}\right)^{1 / 2} .
$$

The corresponding intersection points of $Q$ with $K$ will be the points

$$
A_{i}\left(\xi_{1}=u_{i}+\frac{1}{4 !} k u_{i}^{3}, \xi_{2}=\frac{1}{2} u_{i}^{2}, \xi_{3}=\frac{1}{3 !} u_{i}^{3}\right) \quad(i=1,2) .
$$

The straight line which connects $A_{1}$ and $A_{2}$ cuts the osculating 
plane in the point $\xi_{1}=\xi_{3}=0, \xi_{2}=3 k^{-1}$. That gives the following new characterization of the affine principal normal: the affine principal normal is the line which connects the point $x_{0}$ of the curve with the intersection point of the osculating plane with the straight line $A_{1} A_{2}$.

The osculating plane of $Q$ at $A_{i}$, taking into account (4.1), is

$$
6 u_{i}^{2} \xi_{1}-12 u_{i} \xi_{2}+3 \xi_{3}-2 u_{i}^{3}=0
$$

and it cuts the edges of the affine fundamental trihedral in the following points

$$
y_{1}=x_{0}+\frac{1}{3} u_{i} x_{0}^{\prime}, \quad y_{2}=x_{0}-\frac{1}{6} u_{i}^{2} x_{0}^{\prime \prime}, \quad y_{3}=x_{0}+\frac{2}{3} u_{i}^{3} x_{0}^{\prime \prime \prime} .
$$

The absolute value of the volume of the tetrahedron whose vertices are the points $x_{0}, y_{1}, y_{2}, y_{3}$, taking into account (4.1) and (1.2), is

$$
V=\frac{1}{6}\left(y_{1}-x_{0}, y_{2}-x_{0}, y_{3}-x_{0}\right)=\frac{4}{3} k^{-3} \text {. }
$$

Then we have the theorem:

Let us consider the points $A_{i}(i=1,2)$ in which the osculating cubical parabola $Q$ intersects the cone $K$. The osculating plane of $Q$ at any one of the points $A_{i}$ determines with the affine fundamental trihedral a tetrahedron whose volume $V$ is related to the affine curvature $k$ by (4.5).

5. Geometrical interpretation of the affine torsion. We now give a geometrical interpretation of the affine torsion $t$. For this purpose let us seek the intersection line of two consecutive affine normal planes. The vector equation of the affine normal plane of $C$ at $x_{0}$ is

$$
y(\lambda, \mu)=x_{0}+\lambda x_{0}^{\prime \prime}+\mu x_{0}^{\prime \prime \prime}
$$

and the analogous equation for the affine normal plane at the point $x(s)$, taking into account the relation (1.1), takes the form

$$
\begin{aligned}
\bar{y}(\bar{\lambda}, \bar{\mu})= & x(s)+\bar{\lambda} x^{\prime \prime}(s)+\bar{\mu} x^{\prime \prime \prime}(s) \\
= & x_{0}+\left[(1-t \bar{\mu}) s-\frac{1}{2}\left(t \bar{\lambda}+t^{\prime} \bar{\mu}\right) s^{2}+\cdots\right] x_{0}^{\prime} \\
& +\left[\bar{\lambda}-k \bar{\mu} s+\frac{1}{2}\left(1-k \bar{\lambda}-\left(k^{\prime}+t\right) \bar{\mu}\right) s^{2}+\cdots\right] x_{0}^{\prime \prime} \\
& +\left[\bar{\mu}+\bar{\lambda} s-\frac{1}{2} k \bar{\mu} s^{2}+\cdots\right] x_{0}{ }^{\prime \prime \prime} .
\end{aligned}
$$


In order to find the intersection line of the planes (5.1) and (5.2) we must write

$$
\begin{array}{r}
1-t \bar{\mu}-\frac{1}{2}\left(t \bar{\lambda}+t^{\prime} \bar{\mu}\right) s+\cdots=0, \\
\bar{\lambda}-k \bar{\mu} s+\frac{1}{2}\left(1-k \bar{\lambda}-\left(k^{\prime}+t\right) \bar{\mu}\right) s^{2}+\cdots=\lambda, \\
\bar{\mu}+\bar{\lambda} s-\frac{1}{2} k \bar{\mu} s^{2}+\cdots=\mu
\end{array}
$$

and by elimination of $\bar{\lambda}$ and $\bar{\mu}$ we find

$$
(1-\mu t)+\frac{1}{2}\left(\lambda t+\mu t^{\prime}\right) s+\cdots=0 .
$$

For $s=0$ we have $\mu=1 / t$; hence the intersection line of two consecutive affine normal planes is the line $y(\lambda)=x_{0}+\lambda x_{0}^{\prime \prime}+t^{-1} x_{0}^{\prime \prime}$. Then, the intersection point of the affine binormal at the point $x_{0}$ with the consecutive affine normal plane or, what is the same, the intersection point of the affine binormal with the developable surface enveloped by the affine normal planes, is the point $B \equiv x_{0}+t^{-1} x_{0}^{\prime \prime}$.

Let us now trace through the point $B$ the parallel line to the tangent of $C$ at the point $x_{0}$; if we call $E$ its intersection point with the quadric cone $K$ we find that it is

$$
E \equiv x_{0}+\frac{1}{2} k t^{-1} x_{0}^{\prime}+t^{-1} x_{0}^{\prime} .
$$

Taking into account (1.2) for the volume of the tetrahedron whose vertices are the points $x_{0}, B, A_{1}$ (or $A_{2}$ ) and $E$ we have the expression

$$
V^{*}=\frac{1}{6}\left|\begin{array}{llll}
0 & 0 & 0 & 1 \\
0 & 0 & t^{-1} & 1 \\
\xi_{1} & \xi_{2} & \xi_{3} & 1 \\
\frac{1}{2} k t^{-1} & 0 & t^{-1} & 1
\end{array}\right|
$$

where the values of $\xi_{i}(i=1,2,3)$ are given by (4.3) and (4.2). Consequently we have

$$
V^{*}=\frac{1}{4} t^{-2}
$$

Hence we have obtained the following geometrical interpretation for the affine torsion $t$ of a space curve: 
Through the intersection point $B$ of the affine binormal at the point $x_{0}$ of a space curve $C$ with the developable surface enveloped by the affine normal planes, we trace the parallel line to the tangent of $C$ at $x_{0}$. Let $E$ be the point in which this parallel line intersects the quadric cone $K$. If $A_{i}$ is any one of the points in which the osculating cubical parabola $Q$ intersects the cone $K$, the volume of the tetrahedron whose vertices are the points $x_{0}, B, A_{i}, E$ is related to the affine torsion of $C$ at $x_{0}$ by the relation (5.3).

\section{REFERENCES}

1. W. Blaschke, Vorlesungen ïber Differentialgeometrie, vol. 2, Berlin, 1923.

2. W. Haack, Eine geometrische Deutung der Affin-Invarianten einer Raumkurve, Math. Zeit. vol. 38 (1934) pp. 155-162.

3. E. Salkowski, Affine Differentialgeometrie, Berlin and Leipzig, 1934.

UnIVERSIDAd NACIONAL DEL Litoral, Rosario 